\title{
Neutron Matter Wave Quantum Optics
}

\author{
Helmut Rauch
}

Received: 14 February 2011 / Accepted: 8 May 2011 / Published online: 25 May 2011

(C) The Author(s) 2011. This article is published with open access at Springerlink.com

\begin{abstract}
Neutron matter-wave optics provides the basis for new quantum experiments and a step towards applications of quantum phenomena. Most experiments have been performed with a perfect crystal neutron interferometer where widely separated coherent beams can be manipulated individually. Various geometric phases have been measured and their robustness against fluctuation effects has been proven, which may become a useful property for advanced quantum communication. Quantum contextuality for single particle systems shows that quantum correlations are to some extent more demanding than classical ones. In this case entanglement between external and internal degrees of freedom offers new insights into basic laws of quantum physics. Non-contextuality hidden variable theories can be rejected by arguments based on the Kochen-Specker theorem.
\end{abstract}

Keywords Neutron interferometry · Neutron optics · Quantum experiments · Contextuality $\cdot$ Kochen-Specker $\cdot$ Hadron interferometry

\section{Introduction}

Quantum physics describes nature by means of the Schrödinger equation

$$
i h \frac{\partial|\psi\rangle}{\partial t}=\mathscr{H}|\psi\rangle
$$

where $\mathscr{H}$ denotes the interaction Hamiltonian between physical entities, e.g. particlelike neutrons, with the nuclei of a target. For elastic scattering processes at low ener-

H. Rauch ( $\varangle)$

Atominstitut, Vienna University of Technology, Stadionallee 2, 1020 Wien, Austria

e-mail: rauch@ati.ac.at 
gies a solution of this equation can be given in the form [1]:

$$
\psi=e^{i k z}-b \frac{e^{i k r}}{r}
$$

where the scattering length $b$ is a constant related to the phase shift $\delta_{0}$ between the incident plane and reflected spherical wave, $b=\lim _{k \rightarrow 0}-\left(\sin \delta_{0} / k\right) \cong-\delta_{0} / k$. Since there is yet no complete theory of strong interaction the phase shifts have to be taken as parameters describing the individual interaction. Isotropic scattering can be described within the first Born approximation by the scattering from a point-like potential in form of a Fermi $\delta$-potential

$$
V(\vec{r})=\frac{2 \pi h^{2}}{m} b \delta(\vec{r}) .
$$

In a more general sense the incident wave has to be described by a wave packet which is the general solution of (1) for the interaction free region

$$
\psi(\vec{r}, t)=(2 \pi)^{-3 / 2} \int a(\vec{k}, \omega) e^{i(\vec{k} \vec{r}-\omega t)} d^{3} \vec{k} d \omega .
$$

It represents the coherent superposition of plane waves and, in terms of quantum optics, a multimode coherent state that can be seen as a quasi-classical state. $|a(k, \omega)|^{2}=g(k, \omega)$ denotes the density of states.

The auto-correlation function of the wave function gives the coherence function [2] $\left(\vec{\Delta}=\vec{r}-\vec{r}^{\prime}, \tau=t-t^{\prime}\right)$

$$
\begin{aligned}
\Gamma^{(1)}(\Delta, \tau) & =\left\langle\psi^{*}(\vec{r}, t) \psi\left(\vec{r}^{\prime}, t^{\prime}\right)\right\rangle \\
& =\int g(k, \omega) e^{i\left(\vec{k} \vec{\Delta}-\omega_{k} \tau\right)} d^{3} k d \omega .
\end{aligned}
$$

For a stationary beam this relation simplifies to (Cittert-Zernike theorem)

$$
\Gamma^{(1)}(\vec{\Delta})=\int g(\vec{k}) e^{i \vec{k} \vec{\Delta}} d \vec{k} .
$$

The characteristic dimension of this function defines the coherence length

$$
\Delta_{c}^{2}=\frac{\int \Delta^{2}\left|\Gamma^{(1)}(\Delta)\right| d \Delta}{\int\left|\Gamma^{(1)}(\Delta)\right| d \Delta}
$$

Since the wavelength, $\lambda$, and especially the coherence length of the wave packets are considerably larger than the interatomic distances in condensed matter a simultaneous interaction with many nuclei takes place. This justifies the definition of a coherent $b_{c}$ and an incoherent $b_{i}$ scattering length which are related to a mean phase shift $\left\langle\delta_{0}\right\rangle$ and to its variance $\left\langle\delta_{0}^{2}\right\rangle-\left\langle\delta_{0}\right\rangle^{2}$.

$$
b_{c} \cong-\frac{\left\langle\delta_{0}\right\rangle}{k}
$$




$$
b_{i}^{2} \cong-\frac{\left\langle\delta_{0}^{2}\right\rangle-\left\langle\delta_{0}\right\rangle^{2}}{k^{2}} .
$$

Incoherence can be caused by spin-dependent interaction, by isotopic mixtures. Since the neutron wave interacts with many nuclei a mean interaction potential can be defined (optical potential, i.e. [3])

$$
\bar{V}=\int \frac{2 \pi h^{2}}{m} b_{c} \delta(\vec{r}) d r=\frac{2 \pi h^{2}}{m} b_{c} N,
$$

where $N$ denotes the number of target atoms within the unit volume. Thus, for a stationary situation the time-independent Schrödinger equation has to be solved for a step potential

$$
-\frac{h^{2}}{2 m} \nabla^{2} \psi(r)+\bar{V}(r) \psi(r)=E \psi,
$$

where $E$ denotes the energy-eigenvalue of the motion which has a continuous spectrum when a wave packet is considered $E=h^{2} k^{2} / 2 m$. Inside the material the wave number becomes $K^{2}=2 m[E-\bar{V}(r)] / h^{2}$ and, therefore, an index of refraction can be defined

$$
n=\frac{K}{k}=\sqrt{1-\frac{\bar{V}}{E}} \cong 1-\frac{\bar{V}}{2 E}=1-\lambda^{2} \frac{N b_{c}}{2 \pi},
$$

where the second part of this equation follows from $\bar{V} \ll E$. From this a phase shift, $\chi$, between a wave transmitted through a material of thickness $D$ and a free traveling wave can be derived

$$
\chi=\vec{\Delta} \cdot \vec{k}=(k-K) D=k(1-n) D=-N b_{c} \lambda D .
$$

In a rather general sense the phase shift can be calculated by a path integral along a loop [4]

$$
\chi=\oint k d s
$$

where $k$ denotes the canonical momentum of the neutron.

Similarly a phase shift due to static magnetic interaction $\bar{V}_{m a g}=-\vec{\mu}_{n} \vec{B}(\vec{r})$ can be defined which reads for a homogeneous field $B$ as, e.g. [5]

$$
\chi_{m}= \pm 2 \pi \lambda m \mu B D / h^{2},
$$

and which is just half of the Larmor precession angle indicating its connection to spinor properties (see Sect. 3.1).

Gravitational and Coriolis interaction also cause a phase shift due to the related interactions; $\bar{V}_{\text {grav }}=m \vec{g} \vec{r}$, where $\vec{g}$ denotes the gravitational acceleration and $\bar{V}_{c o r}=-h \Omega(\vec{r} \times \vec{k})$, where $\Omega$ is the Earth rotation frequency. The related phase shifts between the two beams in an interferometer read as: (e.g. [6])

$$
\chi_{\text {grav }}=-2 \pi \lambda m^{2} g A_{0} \sin \alpha / h^{2}+4 \pi m \Omega A_{0} \cos \Theta_{L} \cos \alpha / h .
$$


Fig. 1 Various types of silicon perfect crystal neutron interferometers

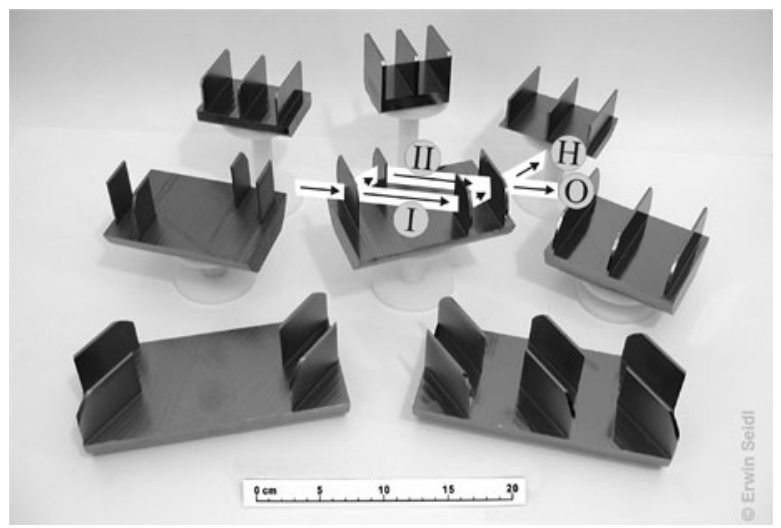

$A_{0}$ denotes the area encircled by the beams, $\alpha$ is the angle of this area against horizontality, and $\Theta_{L}$ is the colatitude angle where the experiment is carried out.

\section{Neutron Interferometry}

Perfect crystal neutron interferometry [5, 7] allows application of different interactions to the widely separated coherent beams and, therefore, the realization of many basic quantum experiments where quantum phases and quantum topology can be studied in detail. Figure 1 shows different perfect crystal neutron interferometers used in the course of our experiments. The perfect arrangement of the atoms within a perfect silicon crystal allows the calculation of wave functions behind the individual plates and behind the whole interferometer. This calculation is based on the dynamical diffraction theory first developed for X-rays and electrons and later on adapted to neutrons $[3,8]$. The related wave functions have quite a complicated structure due to the mutual interferences of internal wave fields, but for the use of such interferometers for quantum measurements the following relations can explain the action of such a device. The wave function behind the interferometer in the forward (0) direction is composed of wave functions arising from beam paths I and II

$$
I \propto\left|\psi_{\mathrm{I}}+\psi_{\mathrm{II}}\right|^{2}
$$

where beam path I is transmitted-reflected-reflected and beam path II is reflectedreflected-transmitted, respectively. Due to symmetry relations both wave functions have to be equal concerning amplitude and phase, $\psi_{\mathrm{I}}=\psi_{\mathrm{II}}$. When a phase shift, $\chi$, is applied to one beam one gets

$$
I \propto\left|\psi_{\mathrm{I}}\right|^{2}(1+\cos \chi)
$$

Small deviations from the perfectness of such a system are unavoidable; small variations of the lattice constant, small rotations of the lattice planes, small temperature gradients, and small vibrations. 

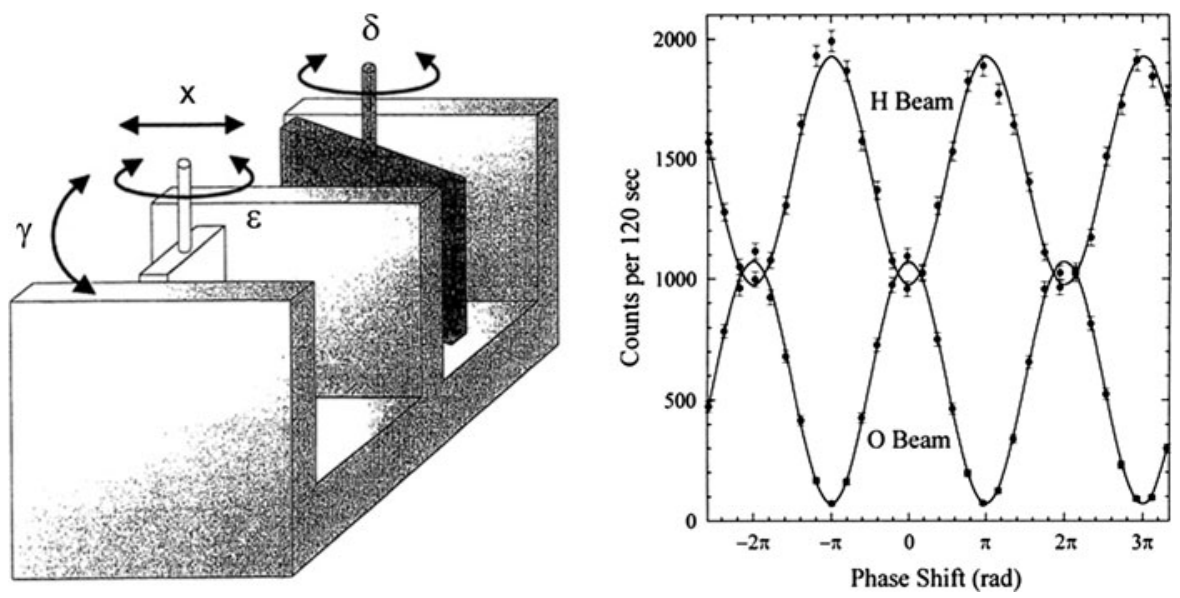

Fig. 2 Arrangement of phase shifters within the interferometer and observed interference pattern

Therefore, the observed interference pattern has to be written as

$$
I=A+B \cos \left(\chi+\chi_{0}\right)
$$

where $A, B$ and $\chi_{0}$ are internal parameters of the individual set-up. Figure 2 shows that a nearly ideal situation can be achieved when the adjustment procedure is done properly. Differently shaped crystals permit interferometers with rather long beam paths $(20 \mathrm{~cm})$, large beam separations $(8 \mathrm{~cm})$ and double loop interferometer set-ups (Fig. 1).

Most of the experiments have been performed with thermal neutrons having an energy of about $0.025 \mathrm{eV}$, a wavelength of about $1.8 \AA$, and an energy spread of $\delta E / E \cong 0.01$. Fission neutrons are slowed down within a moderator of a reactor and a thermal beam is extracted and monochromatized afterwards. All the experiments are done in the single particle regime since the phase space density of any neutron sources is rather small $\left(10^{-15}\right)$, which means that only one neutron at a time is within the apparatus and the next one is still in the uranium nucleus of the reactor fuel. Even so, the results indicate that each neutron behind the interferometer has information about the physical situation in both widely separated beam paths. The arrangement clearly demonstrates wave-particle duality since the neutron after fission and during moderation behaves as particle, inside the interferometer as a wave and behind it, in the detector, as a particle again. This makes such experiments to a playground for the wave-particle dualism debate (e.g. [9]).

\section{Classical Results}

\subsection{Spinor Symmetry: Neutrons in a Magnetic Field}

The interaction Hamiltonian can be written as

$$
\mathscr{H}_{\text {mag }}=-\vec{\mu}_{n} \vec{B}(\vec{r}, t),
$$


Fig. 3 Schematic view of the $4 \pi$-symmetry

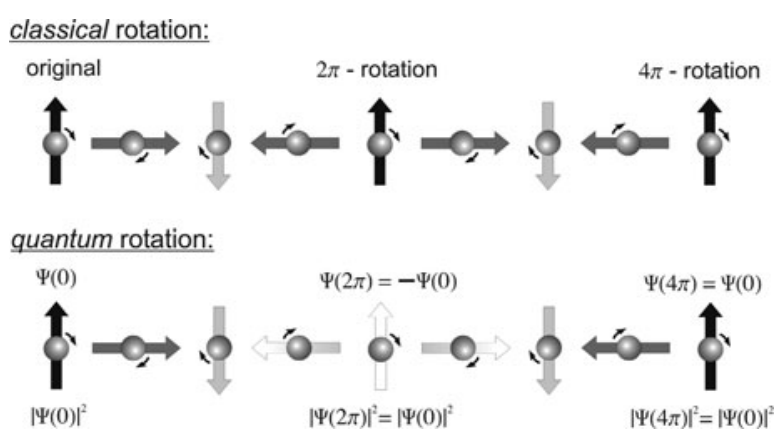

which for a static and homogeneous field simplifies to

$$
\mathscr{H}_{\text {mag }}=-\vec{\mu}_{n} \vec{B} \sigma_{z}
$$

and the neutron wave function evolves as

$$
\begin{aligned}
|\psi(t)\rangle & =e^{-i H t / h}|\psi(0)\rangle \\
& =e^{-\mu_{n} B \sigma_{z} / h}|\psi(0)\rangle=e^{-i \sigma_{z} \alpha / 2}|\psi(0)\rangle=|\psi(\alpha)\rangle,
\end{aligned}
$$

where $\alpha$ denotes the Larmor precession angle

$$
\alpha=\frac{2 \mu_{n} B t}{h}=\frac{2 \mu_{n} B L}{h v},
$$

where $L$ is the length of the interaction region and $v$ is the velocity of the neutrons. From relation (28) follows

$$
\begin{aligned}
& |\psi(2 \pi)\rangle=-|\psi(0)\rangle, \\
& |\psi(4 \pi)\rangle=|\psi(0)\rangle,
\end{aligned}
$$

showing the $4 \pi$-symmetry of spinor wave functions (Fig. 3).

This feature becomes measurable with the neutron interferometer as predicted by Aharonov and Susskind [10] and by Bernstein [11]

$$
\left.I_{0} \propto \| \psi_{0}(0)\right\rangle+\left.\left|\psi_{0}(\alpha)\right\rangle\right|^{2} \propto 1+\cos \frac{\alpha}{2} .
$$

The related experiments have been done nearly simultaneously by our group [12] and by a U.S. one [13] and demonstrated this basic feature (Fig. 4). It shows the experimental set-up and the first results giving $\alpha=(715.9 \pm 3.8)^{\circ}$. It has been measured with unpolarized and with polarized neutrons which demonstrates the intrinsic feature of this phenomenon. Depending on the axis of quantization chosen the effect can be described by the index of refraction or a rotation around the magnetic field. Since the effect depends on the strength of the field the related phase shift has to be seen as a dynamical one in comparison with a geometric phase as discussed in Sect. 4.1. 

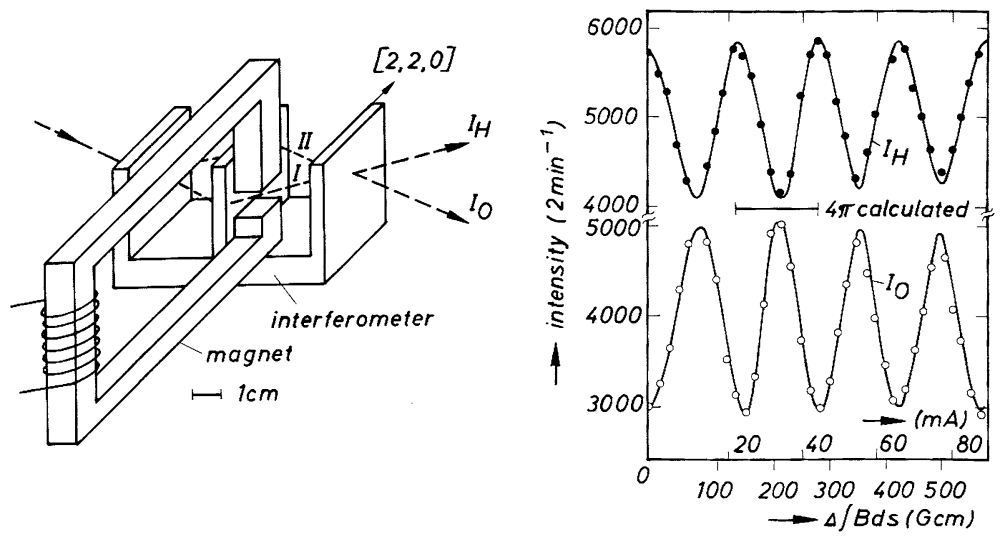

Fig. 4 Experimental set-up and results of the first verification experiment of $4 \pi$-symmetry of spinor wave functions [12]

Fig. 5 Sketch of quantum and classical spin-superposition

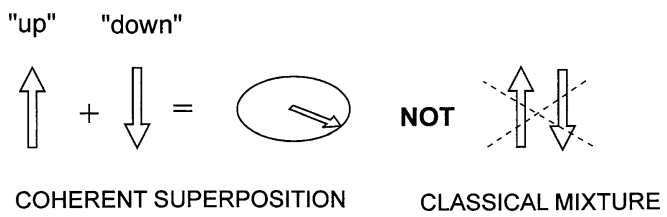

\subsection{Spin Superposition}

In a famous article on the theory of measurement Wigner [14] brought attention to the curious situation of a superposition of a spin-up and a spin-down state since quantum mechanics predicts a state perpendicular to both initial states with an angle depending on the relative phase of those states, whereas classical physics predicts a mixture (Fig. 5).

When one uses polarized incident neutrons and rotates the direction of polarization in one beam path by $\pi$ the related wave function reads

$$
\left|\psi^{\prime}(\chi, \pi)\right\rangle=e^{i \chi} e^{-i \sigma_{y} \pi / 2}|z\rangle=e^{i \chi}|-z\rangle,
$$

and the final polarization after superposition becomes $\left(\psi=\psi^{\prime}+\psi^{\prime \prime}\right)$

$$
\vec{P}=\frac{\langle\psi|\vec{\sigma}| \psi\rangle}{\langle\psi \mid \psi\rangle}=\left(\begin{array}{c}
\cos \chi \\
\sin \chi \\
0
\end{array}\right) .
$$

It shows that an initially pure state in the $|z\rangle$-direction is transferred to a pure state in the $x, y$-plane indicating again that information from both beam parts are needed to explain these phenomena. The scheme and the results of such an experiment are shown in Fig. 6 [15]. The spin rotation in this case achieved with by Larmor precession coils [16].

A similar experiment has been done with a resonance Rabi-flipper [17]. In this case, the spin reversal is caused by a time-dependent interaction and is associated with 

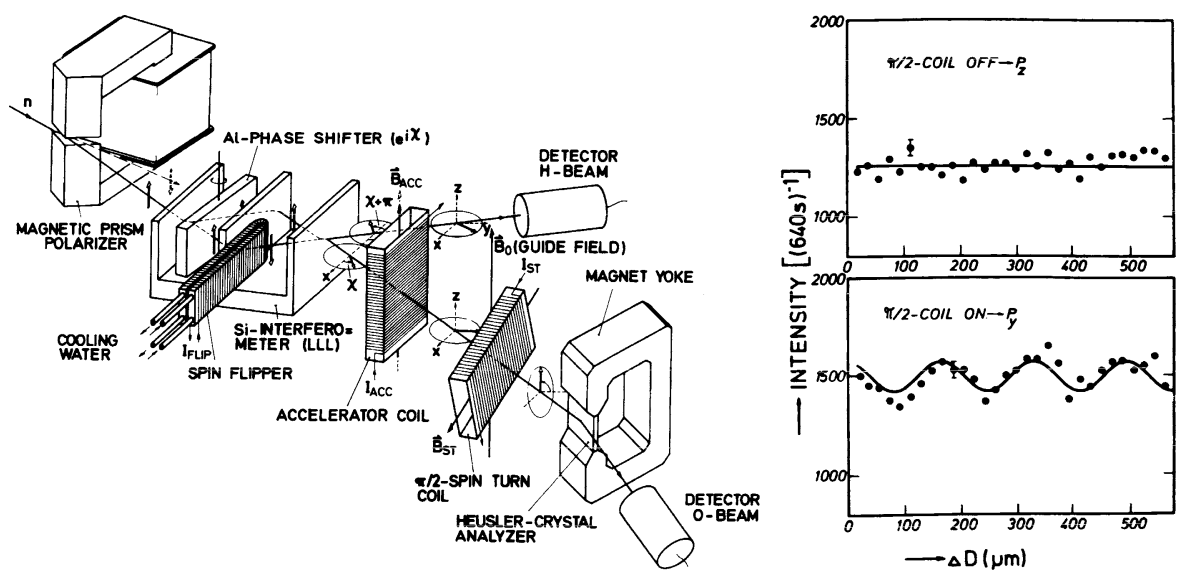

Fig. 6 Experimental arrangement and results of the static spin-superposition experiment [15]
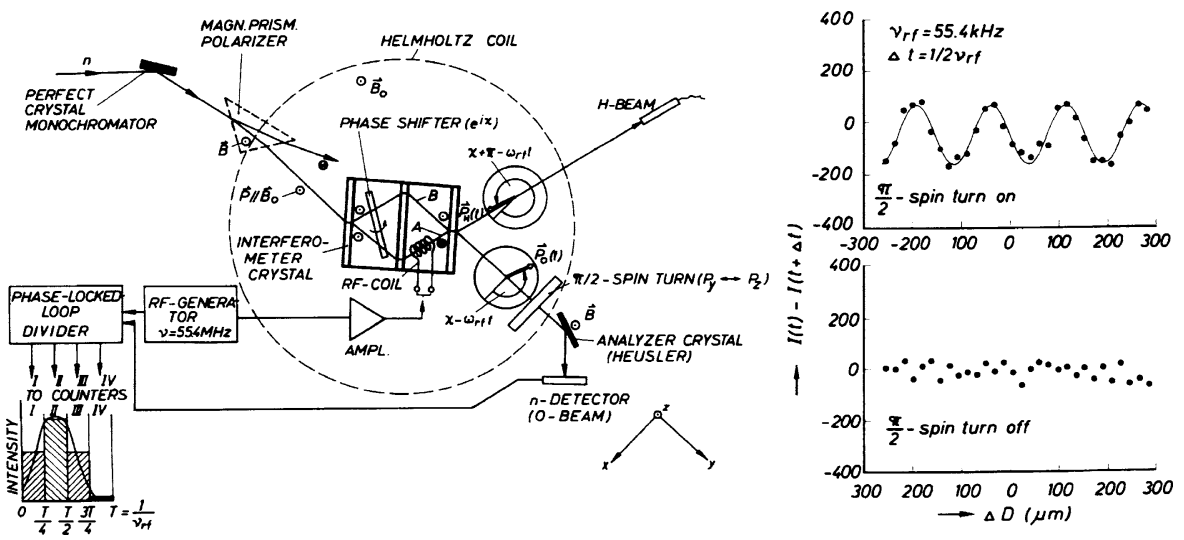

Fig. 7 Experimental arrangement and results of the dynamic spin-superposition experiment indicating the stroboscopic measuring technique [20]

an energy change of $h \omega_{r}=2|\mu| B_{0}[18,19]$. Therefore, the wave function changes as:

$$
\psi^{\prime \prime}\left(\chi, \omega_{r}\right)=e^{i \chi} e^{-i\left(\omega-\omega_{r}\right) t}|-z\rangle,
$$

and the final polarization rotates within the $x, y$-phase as

$$
\vec{P}=\left(\begin{array}{c}
\cos \left(\chi-\omega_{r} t\right) \\
\sin \left(\chi-\omega_{r} t\right) \\
0
\end{array}\right) .
$$

which has been verified experimentally (Fig. 7 [20]). Since one photon is exchanged between the neutron and the resonator, the question arises whether this can be used 

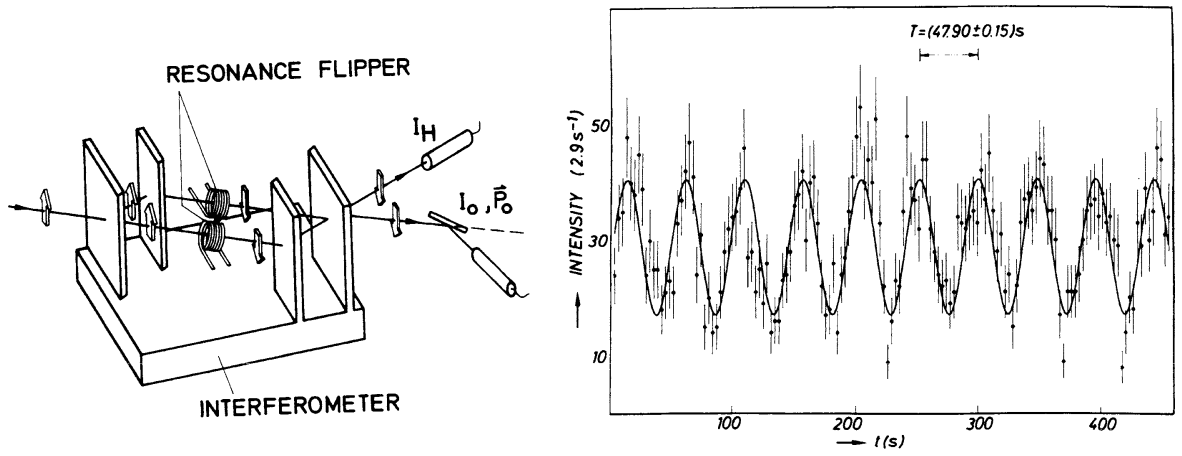

Fig. 8 Double coil experiment verifying the magnetic Josephson effect [25]

for beam path detection in the sense of Feynman's discussion of the double slit experiment [21].

It can be concluded "no" because phase information, $\varphi$, of the classical resonance field is needed to perform the stroboscopic registration of neutrons and then it follows from the particle number-phase uncertainty relation in its simplest form $\Delta N \Delta \varphi \geq 1 / 2$ (e.g. [22]) that a single exchange photon cannot be registered. For a more profound discussion see [23, 24].

\subsection{Magnetic Josephson Effect}

When two resonance flippers are inserted, one in each beam, and operated at a slightly different frequencies the wave function becomes

$$
\psi=\psi_{0}^{\mathrm{I}}+\psi_{0}^{\mathrm{II}}=e^{-i\left(\omega-\omega_{r 1}\right) t}|-z\rangle+e^{i \chi} e^{i\left(\omega-\omega_{r 2}\right) t}|-z\rangle,
$$

which yields an intensity modulation as

$$
I_{0} \propto 1+\cos \left[\chi+\left(\omega_{r 1}-\omega_{r 2}\right) t\right] .
$$

Thus the intensity oscillates between the forward (0) and the diffracted beam $(\mathrm{H})$ being driven by an extremely small frequency difference which relates in the case of our measurements to a small energy difference, $\Delta E=8.6 \times 10^{-17} \mathrm{eV}$ (Fig. 8) [25]. A similar experiment has also been done by means of a neutron polarimeter set-up by a Japanese group [26].

One can formulate the different energy transfer as a time-dependent phase shift

$$
\Delta=\Delta_{1}-\Delta_{2}=\left(\omega_{r 1}-\omega_{r 2}\right) t=\frac{2 \mu_{n} \Delta B_{0}}{h} t,
$$

which compares to the analog equation for the electric Josephson effect

$$
\phi=\phi_{2}-\phi_{1}=\frac{2 e V}{h} t
$$

where the rapidly oscillating tunneling current $\left(I_{s}=I_{\text {Max }} \sin \phi\right)$ is driven by the electric potential between the tunnel junction [27]. 
When an off-resonance field is applied multi-photon exchange occurs which is related to dressed neutron phenomena. This behavior has been tested in a dedicated experiment [28].

\section{Recent Experiments}

\subsection{Geometric Phases}

The solution of the Schrödinger equation (1) for an adiabatic evolution can be written in the form

$$
|\psi(t)\rangle=e^{i \phi(t)}|n(\vec{R}(t))\rangle
$$

where $|n(\vec{R}(t))\rangle$ denotes the eigenstate of the instantaneous Hamiltonian $\mathscr{H}(\vec{R}(t))|n(\vec{R}(t))\rangle=E_{n}(t)|n(\vec{R}(t))\rangle$ and $\phi(t)$ a generalized phase. Inserting this equation into (1) and integrating over a closed path $C$ in parameter space $|\psi(\vec{R}(T))\rangle=|\psi(\vec{R}(0))\rangle$ one gets a separation into a dynamical phase $\left(\phi_{d}\right)$ accumulating the energy (momentum) change along the loop and a geometric phase $\left(\phi_{g}\right)$ which is independent from energy [29]. For a constant magnetic field in one direction only a dynamical phase exists, as shown in the previous chapters.

$$
\begin{aligned}
\phi(T)= & \arg \langle\psi(T) \mid \psi(0)\rangle=-\frac{1}{h} \int_{0}^{T} E_{n}(\vec{R}(t)) d t \\
& +i \oint d \vec{R}\left\langle n(\vec{R})\left|\vec{\nabla}_{R}\right| n(\vec{R})\right\rangle=\phi_{d}+\phi_{g} .
\end{aligned}
$$

In the case of a slow change of the Hamiltonian (magnetic field) which corresponds to an adiabatic evolution the neutron spin will be pinned to the direction of the magnetic field

$$
\vec{B}(t)=B \vec{n}(t)=B\left(\begin{array}{c}
\cos \phi(t) \sin \Theta(t) \\
\sin \phi(t) \sin \Theta(t) \\
\cos \Theta(t)
\end{array}\right)
$$

with the eigenvectors

$$
\begin{aligned}
& \left|\psi_{\uparrow}(\Theta, \phi)\right\rangle=\left(\begin{array}{c}
\cos \frac{\Theta(t)}{2} \\
e^{i \phi(t)} \sin \frac{\Theta(t)}{2}
\end{array}\right), \\
& \left|\psi_{\downarrow}(\Theta, \phi)\right\rangle=\left(\begin{array}{c}
\sin \frac{\Theta(t)}{2} \\
-e^{i \phi(t)} \cos \frac{\Theta(t)}{2}
\end{array}\right),
\end{aligned}
$$

which yields

$$
\left\langle\psi_{\uparrow}\left|\frac{\partial}{\partial \phi}\right| \psi_{\uparrow}\right\rangle=\frac{i}{2}(1-\cos \Theta(t)),
$$


Fig. 9 Sketch of a non-adiabatic (left) and a non-adiabatic and non-cyclic evolution (right) of a quantum system and indication of the related geometric phases

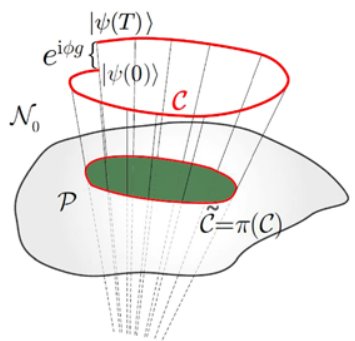

and when we move along a circle of latitude $(\Theta=$ constant $)$ we obtain the well-known Berry phase

$$
\phi_{g}=i \int_{0}^{2 \pi} \frac{i}{2}(1-\cos \Theta(t)) d \phi=-\pi(1-\cos \Theta)=-\Omega / 2,
$$

i.e., the geometric phase is just half of the solid angle $\Omega$ enclosed by the path. A related neutron interferometric experiment has been performed by Wagh et al. [30] with Larmor precession coils rotated in an opposite sense in both coherent beams. Complete agreement between theory and experiment has been achieved.

Aharonov and Anandan [31] generalized this approach to any cyclic evolution of a quantum system (e.g. [32]). They found that any excursion curve in Hilbert space (C) having the same projections onto $\mathcal{P}$ have the same geometric phase modular $2 \pi$ (Fig. 9)

$$
\phi_{g}=2 \pi n+i \int_{0}^{T}\left\langle\psi(t)\left|\frac{d}{d t}\right| \psi(t)\right\rangle d t .
$$

Later on Samuel and Bhandari [33] generalized this formalism to non-cyclic and non-adiabatic evolutions. This formalism is strongly based on the centennial work of Pancharatnam [34]. In this case, a geodesic connection between $|\psi(0)\rangle$ and $|\psi(t)\rangle$ has to be drawn (Fig. 9). This means that the shortest possible path has to be chosen to define the enclosed area. When the initial and final states are orthogonal to each other a special treatment is necessary $[35,37]$.

A related experiment has been performed with a double loop interferometer where two phase shifters (PS) and an absorber (A) permit quite peculiar state excursions (Fig. 10) [36].

The upper beam $\left|\psi_{t}^{0}\right\rangle$ of the first loop is used as a reference beam with adjustable phase $\eta$ and as the incident wave for loop 2 where the transmitted beam, $|p\rangle$, becomes attenuated $\left(T=\exp \left(-\sigma_{t} N D\right)\right)$ and phase shifted $\left(\chi_{2}\right)$ and the orthogonal beam, $\left|p^{\perp}\right\rangle$ which becomes phase shifted by $\chi_{1}$. This gives an overlap of the reference beam $\left|\psi_{\text {ref }}\right\rangle$ and the loop 2 beam $\left|\psi_{2}\right\rangle$

$$
\begin{aligned}
& \left|\psi_{r e f}\right\rangle \propto\left(|p\rangle+\left|p^{\perp}\right\rangle\right)=|q\rangle, \\
& \left|\psi_{2}\right\rangle \propto\left(e^{i \chi_{1}}+\sqrt{T} e^{i \chi_{2}}\right)|q\rangle, \\
& \phi_{d}=\arg \left\langle\psi_{r e f} \mid \psi_{2}\right\rangle=\frac{\chi_{1}+\chi_{2}}{2} \operatorname{arctg}\left[\frac{\chi_{2}-\chi_{1}}{2}\left(\frac{1-\sqrt{T}}{1+\sqrt{T}}\right)\right] .
\end{aligned}
$$




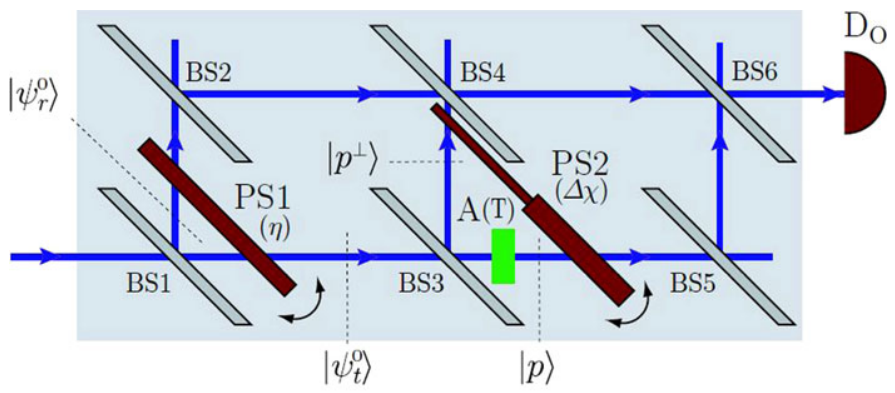

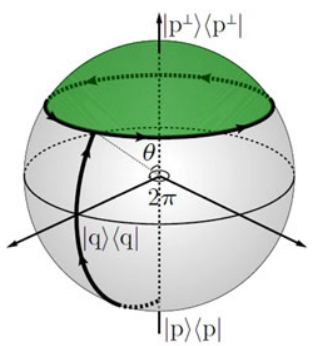

(a) Cyclic evolution

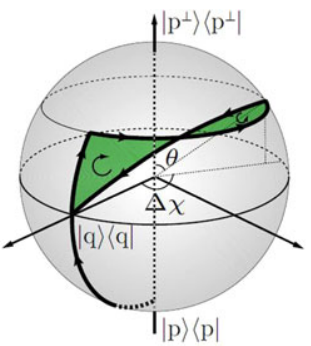

(b) Non-cyclic evolution

Fig. 10 Sketch of a two-loop interferometer (above) and two examples of quantum evolutions to measure the geometric phase (left) and a non-cyclic phase (right) [36]

Since the dynamical phase can be written as

$$
\phi_{d}=\frac{\chi_{1}+T \chi_{2}}{1+T},
$$

which becomes a constant when

$$
\chi_{1}+T \chi_{2}=\text { const. }
$$

A proper manipulation of phase shifters and the absorbers permit cyclic and noncyclic evolutions on the Bloch sphere where the north pole and the south pole correspond to well defined paths along the upper, $\left|p^{\perp}\right\rangle$, and the lower beam paths $|p\rangle$ . within the second interferometer loop. The absorber determines the latitude where the evolution driven by the phase shifter PS2 takes place. This allows us to write the absorption in the form

$$
T=\tan ^{2} \frac{\Theta}{2} .
$$

The geometric phase can be measured when closed cycles at different latitudes are chosen. Non-cyclic evolutions occur when such a rotation is stopped before a cycle is complete and then this endpoint has to be connected by a geodesis line to the equator. Figure 11 shows the results of such measurements [36]. They clearly define the geometric phase and the non-cyclic phase for situations shown in Fig. 10 (right).

The results indicate that geometric phases are well-defined and well-measurable quantities which may become even more important in future since they seem to be 
Fig. 11 Results of the non-cyclic phase experiment according to non-cyclic excursion in Fig. 10 in comparison with calculated values [36]
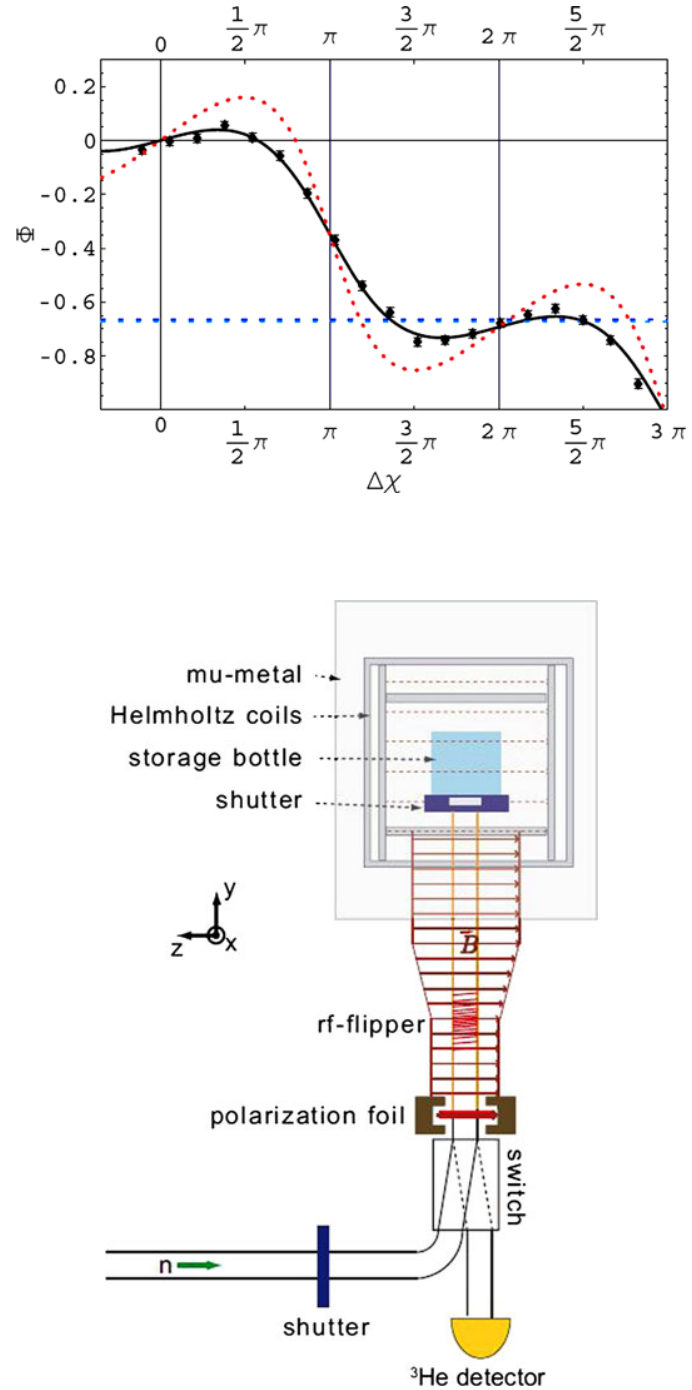

Fig. 12 Experimental set-up to measure geometric phases with ultra-cold neutrons by means of a spin-echo method to balance the dynamical phase [39]

less sensitive to any fluctuation of external parameters [38]. A related experiment with bottled ultra-cold neutrons has been performed recently [39] (Fig. 12).

Polarized ultra-cold neutrons rotate, guided by a magnetic field, around the axis of a Bloch sphere on the northern hemisphere, then they are flipped by $\pi$ and rotate in the opposite direction on the southern hemisphere. This eliminates the dynamical phase and provides direct access to the geometric phase. This is measured without and with additional noisy fields which change slightly the direction but not the strength. This gave results as shown in Fig. 13 [39]. This clearly indicates that the geometric phase becomes better defined when the neutron spends longer time within the noisy field, an effect opposite to the behavior of the dynamical phase. 

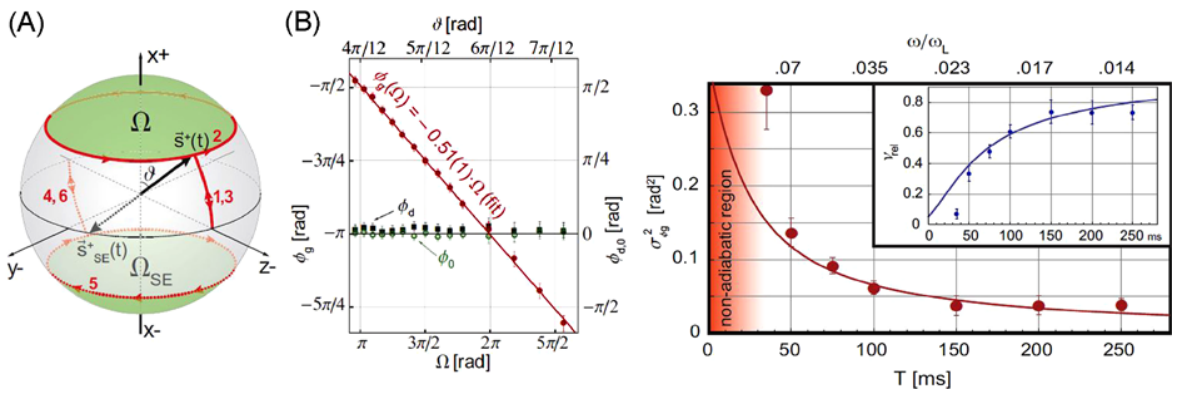

Fig. 13 Experimental situation (left), results for the measurement of the geometric phase (middle), and its stability against magnetic fluctuations (right) [39]

\subsection{Contextuality and Kochen-Specker Phenomenon}

Entanglement of pairs of photons or material particles is a well-known phenomenon [40-43], but entanglement means in a more general sense entanglement of different degrees of freedom. Therefore, entanglement can also exist between different degrees of freedom of a single particle system which yields to quantum contextuality $[44,45]$. Contextuality implies that the outcome of a measurement depends on the experimental context, i.e. the outcome of a previous or simultaneous experiment of another observable [46]. In this respect it is a more stringent demand than non-locality. In a related neutron experiment $[47,48]$ the commuting observables of the spin (s) and the beam path (p) through the interferometer act as two independent degrees of freedom. Both represent a two-level system and can be described by Pauli spin matrices with the commutation relations

$$
\begin{aligned}
& {\left[\sigma_{j}^{s}, \sigma_{k}^{p}\right]=0 \text { for }\{j, k\}=\{x, y\},} \\
& {\left[\sigma_{x}^{s} \sigma_{y}^{p}, \sigma_{y}^{s} \sigma_{x}^{p}\right]=0 .}
\end{aligned}
$$

and, applied to a Bell-like state (57), one obtains the eigenvalue equations

$$
\begin{aligned}
& \sigma_{i}^{s} \sigma_{i}^{p}|\psi\rangle=-|\psi\rangle, \quad i=x, y, \\
& \left(\sigma_{x}^{s} \sigma_{y}^{p}\right)\left(\sigma_{y}^{s} \sigma_{x}^{p}\right)|\psi\rangle=-|\psi\rangle .
\end{aligned}
$$

The related Bell-state can be produced within the interferometer when a polarized incident beam is split coherently into two beam paths (I and II) and the spin in one beam path is rotated by Larmor precession in the $y$ and in the other beam path to the $+y$ direction (Fig. 14).

The entangled Bell-states read as

$$
|\psi\rangle=|\rightarrow\rangle \otimes|\mathrm{I}\rangle \pm|\leftarrow\rangle \otimes|\mathrm{II}\rangle,
$$

and the other three can be formulated similarly. In a full quantum tomographic analysis these Bell-like states have been measured [49]. This shows spin-path entanglement in spin-path joint measurements. In all these cases Bell-like inequalities can be formulated to demarcate a quantum world from a classical one. 


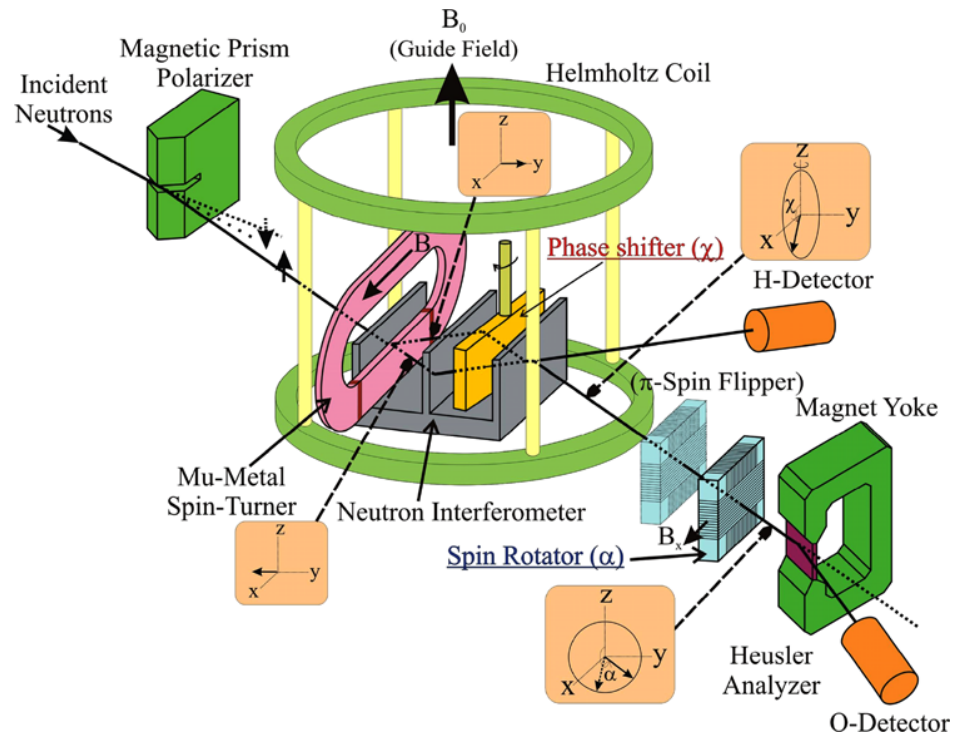

Fig. 14 Experimental arrangement to produce and to analyze spin-path entangled neutron states [45]

The phase shift $\chi$ between the beams and the spin rotation angle $\alpha$ are used as path and spin parameters [47]

$$
\begin{aligned}
& -2 \leq S \leq 2 \quad \text { (classic), } \\
& -2 \sqrt{2} \leq S \leq 2 \sqrt{2} \quad \text { (quantum), } \\
& S=E\left(\alpha_{2}, \chi_{2}\right)+E\left(\alpha_{1}, \chi_{2}\right)-E\left(\alpha_{2}, \chi_{1}\right)+E\left(\alpha_{2}, \chi_{2}\right), \\
& E(\alpha, \chi)=\frac{N(\alpha, \chi)+N(\alpha+\pi, \chi+\pi)-N(\alpha, \chi+\pi)-N(\alpha+\pi, \chi)}{N(\alpha, \chi)+N(\alpha+\pi, \chi+\pi)+N(\alpha, \chi+\pi)+N(\alpha+\pi, \chi)} .
\end{aligned}
$$

The maximal violation towards the quantum mechanical description happens for the following parameters: $\alpha=0, \alpha_{2}=\pi / 2, \chi_{2}=\pi / 4$, and $\chi_{2}=-\pi / 4$. Typical results are shown in Fig. 15.

Careful data analysis gave a value of $S=2.051 \pm 0.019$, i.e. beyond the classical prediction. The reason why this value is considerably below $2 \sqrt{2}$ lies in imperfections of the set-up. The contrast of the interference was about $74 \%$ mainly caused by stray fields of the spin rotator, and the degree of polarization was $95 \%$. Nevertheless quantum contextuality has been demonstrated indicating an intrinsic correlation between the spin and the momentum (path) variables.

A more recent experiment [48] dealt with the Kochen-Specker theorem [44] and the Mermin inequalities [45], where even stronger violations of classical hidden variable theories can be verified. A related test of the Kochen-Specker theorem was formulated by Simon et al. [50] and realized for photons by Huang et al. [51]. A related neutron experiment has been performed using a set-up similar to that shown in Fig. 14 but with the additional feature that the beam paths could be closed alternatively by means of an absorber sheet [48]. The measurement of the product 
Fig. 15 Experimental results of the contextuality experiment with spin-path entangled neutron states [47]

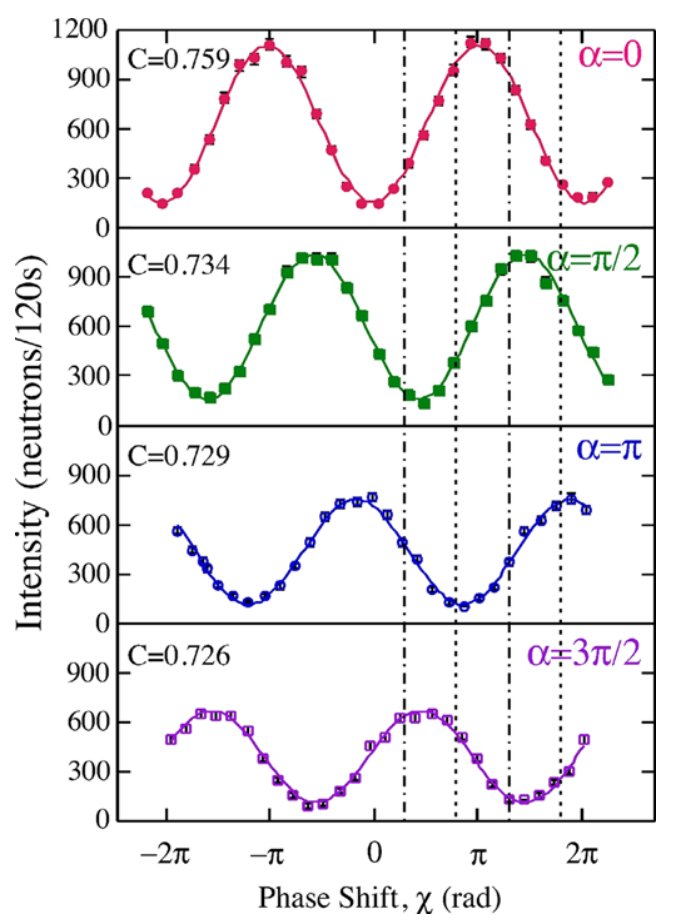

observable $\left(\sigma_{x}^{s} \sigma_{y}^{p}\right)\left(\sigma_{y}^{s} \sigma_{x}^{p}\right)$ was done by measuring $\left(\sigma_{z}^{s} \sigma_{z}^{p}\right)$ and using a priori the non-contextuality relation. The measurable quantity is defined by a sum of product observables

$$
C=\hat{\mathrm{I}}-\sigma_{x}^{s} \sigma_{x}^{p}-\sigma_{y}^{s} \sigma_{y}^{p}-\left(\sigma_{x}^{s} \sigma_{y}^{p}\right)\left(\sigma_{y}^{s} \sigma_{x}^{p}\right) .
$$

In any experiment expectation values only can be measured. For non-contextual models the last term can be separated:

$$
\left\langle\left(\sigma_{x}^{s} \sigma_{y}^{p}\right)\right\rangle\left\langle\left(\sigma_{y}^{s} \sigma_{y}^{p}\right)\right\rangle=\left\langle\sigma_{x}^{s}\right\rangle\left\langle\sigma_{y}^{p}\right\rangle\left\langle\sigma_{y}^{s}\right\rangle\left\langle\sigma_{x}^{p}\right\rangle,
$$

which gives

$$
C_{n c}= \pm 2,
$$

whereas quantum mechanics predicts

$$
C_{q m}=4 .
$$

The measured value was

$$
C_{\text {exp }}=3.138 \pm 0.0115,
$$

which is well above the non-contextuality (classical) limit of 2 and provides an allversus-nothing-type contradiction. It provides a Peres-Mermin proof of quantummechanics against non-contextual hidden variable theories. 
A debate in literature $[50,52]$ criticized the a priori use of the non-contextuality relation $\left(\sigma_{x}^{s} \sigma_{y}^{p}\right) \cdot\left(\sigma_{y}^{s} \sigma_{x}^{p}\right)=\left(\sigma_{z}^{s} \sigma_{z}^{p}\right)$ and in this connection the use of an absorber to measure this quantity. In a follow-up proposal [53] and subsequent experiment [54] the previous result (57) has been verified. In this case a quantum erasure has been used instead of an absorber and, therefore, all quantities required for (57) could be measured within the same context.

Years ago it has been shown that the Zeeman energy $\left(h \omega=2 \mu B_{0}\right)$ can be exchanged between the neutron and a resonance coil coherently [55]. This provides the basis for triple entanglement experiments using spin-path-energy as independent degrees of freedom [56]. These GHZ-states are a new tool for basic neutron quantum optics experiments and may be new components of quantum computing elements like CNOT gates. The energy states have geometric nature and may be rather robust under dissipative effects as shown in Sect. 4.1.

\section{Discussion}

Neutron interferometry is a powerful tool to measure quantum phenomena under new fashion. Single particle interference exhibits all basic features of this fundamental theory and multi-particle systems only complete these insights. Here, a review is given of measurements of various topological phases and their robustness against fluctuations and interactions with the environment. Indeed it appears that geometric phases are much more robust than the dynamical phase and this may have consequences for future quantum information systems. It has also been shown that entanglement between external and internal degrees of freedom in single particle systems can be achieved which leads to quantum contextuality, another important feature of quantum physics. Bell-like inequalities can be formulated and tested with distinct consequences for the understanding of multi-particle entanglement. Noncontextuality hidden variable theories can be turned down by precise measurements of Peres-Mermin inequalities which are based on the Kochen-Specker theorem.

Acknowledgements Most of the experiments described in this review have been financially supported by the Austrian Science Fund (SFB 1513 and P-18943-N2D). Useful cooperation with my colleagues Yuji Hasegawa, Stefan Filipp, Juergen Klepp and Hartmut Lemmel is gratefully acknowledged.

Open Access This article is distributed under the terms of the Creative Commons Attribution Noncommercial License which permits any noncommercial use, distribution, and reproduction in any medium, provided the original author(s) and source are credited.

\section{References}

1. Blatt, J.M., Weisskopf, V.F.: Theoretical Nuclear Physics. Wiley, New York (1932)

2. Glauber, R.J.: Phys. Rev. 130, 2766 (1963)

3. Sears, V.F.: Can. J. Phys. 56, 1261 (1978)

4. Feynman, R.P.: Rev. Mod. Phys. 20, 367 (1948)

5. Rauch, H., Werner, S.A.: Neutron Interferometry. Clarendon, Oxford (2000)

6. Staudenmann, J.L., Werner, S.A., Colella, R., Overhauser, A.W.: Phys. Rev. A 21, 1419 (1980)

7. Rauch, H., Treimer, W., Bonse, U.: Phys. Lett. A 47, 369 (1974) 
8. Rauch, H., Petrascheck, D.: In: Dachs, H. (ed.) Neutron Diffraction. Top. Curr. Phys., vol. 6, p. 303. Springer, Heidelberg (1978)

9. Afshar, S.S., Flores, E., McDonalds, K.F., Kroesel, E.: Found. Phys. 37, 295 (2007)

10. Aharonov, Y., Susskind, L.: Phys. Rev. 158, 1237 (1967)

11. Bernstein, H.J.: Phys. Rev. Lett. 18, 1102 (1967)

12. Rauch, H., Zeilinger, A., Badurek, G., Wilfing, A., Bauspiess, W., Bonse, U.: Phys. Lett. A 54, 425 (1975)

13. Werner, S.A., Colella, R., Overhauser, A.W., Eagen, C.F.: Phys. Rev. Lett. 35, 1053 (1975)

14. Wigner, E.P.: Am. J. Phys. 31, 6 (1963)

15. Summhammer, J., Badurek, G., Rauch, H., Kischko, U.: Phys. Rev. A 27, 2532 (1983)

16. Mezei, F.: Z. Phys. 255, 146 (1972)

17. Rabi, I.I.: Phys. Rev. 51, 652 (1937)

18. Drabkin, G.M., Zhitnikov, R.A.: Sov. Phys. JETP 11, 729 (1960)

19. Alefeld, B., Badurek, G., Rauch, H.: Z. Phys. B, Condens. Matter Quanta 41, 231 (1981)

20. Badurek, G., Rauch, H., Summhammer, J.: Phys. Rev. Lett. 51, 1015 (1983)

21. Feynman, R.P., Leighton, R.B., Sands, K.: The Feynman Lectures on Physics, vol. III. AddisonWesley, Reading (1965)

22. Carruthers, P., Nieto, M.N.: Phys. Rev. Lett. 53, 411 (1968)

23. Namiki, M., Pascazio, S., Nakazato, H.: Decoherence and Quantum Measurement. World Scientific, Singapore (1987)

24. Scully, M.O., Schwinger, J., Walther, H.: Nature 351, 111 (1991)

25. Badurek, G., Rauch, H., Tuppinger, D.: Phys. Rev. A 34, 2600 (1986)

26. Yamazaki, D., Ebisawa, T., Kawai, T., Tasaki, S., Hino, M., Akayoshi, T., Achiwa, H.: Physica B 141-143, 186 (1998)

27. Josephson, B.D.: Rev. Mod. Phys. 46, 251 (1974)

28. Summhammer, J., Hamacher, K.A., Kaiser, H., Weinfurter, H., Jacobson, D.L., Werner, S.A.: Phys. Rev. Lett. 75, 3206 (1995)

29. Berry, M.V.: Proc. R. Soc. Lond. Ser. A, Math. Phys. Sci. A392, 45 (1984)

30. Wagh, A.G., Rakhecha, V.C., Summhammer, J., Badurek, G., Weinfurter, H., Allman, B.E., Kaiser, H., Hamacher, K., Jacobson, D.L., Werner, S.A.: Phys. Rev. Lett. 78, 755 (1997)

31. Aharonov, Y., Anandan, J.S.: Phys. Rev. Lett. 58, 1593 (1987)

32. Tomita, A., Chiao, R.Y.: Phys. Rev. Lett. 57, 937 (1986)

33. Samuel, J., Bhandari, R.: Phys. Rev. Lett. 60, 2339 (1988)

34. Pancharatnam, S.: Proc. Indian Acad. Sci., Sect. A, Phys. Sci. 44, 247 (1956)

35. Bhandari, R.: Phys. Rep. 281, 1 (1997)

36. Filipp, S., Hasegawa, Y., Loidl, R., Rauch, H.: Phys. Rev. A 72, 021602 (2005)

37. Shapere, A., Wilczek, F.: Geometric Phases in Physics. World Scientific, Singapore (1989)

38. De Chiara, G., Palma, G.M.: Phys. Rev. Lett. 91, 090404 (2003)

39. Filipp, S., Klepp, J., Hasegawa, Y., Plonka-Spekr, Ch., Schmidt, U., Geltenbort, P., Rauch, H.: Phys. Rev. Lett. 102, 030404 (2009)

40. Einstein, A., Podolski, B., Rosen, N.: Phys. Rev. 47, 777 (1935)

41. Bell, J.S.: Physics 1, 195 (1964)

42. Clauser, J.F., Shimony, A.: Rep. Prog. Phys. 41, 1978 (1881)

43. Bertlmann, R.A., Zeilinger, A. (eds.): Quantum [Un]speakables. Springer, Berlin (2002)

44. Kochen, S., Specker, F.P.: J. Math. Mech. 17, 59 (1967)

45. Mermin, D.: Phys. Rev. Lett. 65, 1838 (1990)

46. Roy, S.M., Singh, V.: Phys. Rev. A48, 3379 (1993)

47. Hasegawa, Y., Loidl, R., Badurek, G., Baron, M., Rauch, H.: Nature 425, 45 (2003)

48. Hasegawa, Y., Loidl, R., Badurek, G., Baron, M., Rauch, H.: Phys. Rev. Lett. 97, 23041 (2006)

49. Hasegawa, Y., Loidl, R., Badurek, G., Filipp, S., Klepp, J., Rauch, H.: Phys. Rev. A 76, 052108 (2007)

50. Simon, C., Zukovski, M., Weinfurter, H., Zeilinger, A.: Phys. Rev. Lett. 85, 1783 (2000)

51. Huang, Y.-F., Li, C.-F., Zhang, Y.-S., Pan, J.-W., Guo, G.-C.: Phys. Rev. Lett. 90, 250401 (2003)

52. Cinelli, C., Barbieri, M., Perris, R., Mataloni, P., De Martini, F.: Phys. Rev. Lett. 95, 240405 (2005)

53. Cabello, A., Filipp, S., Rauch, H., Hasegawa, Y.: Phys. Rev. Lett. 100, 130404 (2008)

54. Bartosik, H., Klepp, J., Schmitzer, C., Sponar, S., Cabello, A., Rauch, H., Hasegawa, Y.: Phys. Rev. Lett. 103, 040403 (2009)

55. Alefeld, B., Badurek, G., Rauch, H.: Z. Phys. 41, 231 (1981)

56. Sponar, S., Klepp, J., Loidl, R., Filipp, S., Badurek, G., Hasegawa, Y., Rauch, H.: Phys. Rev. A 78, 061604 (2008) 